PANCOTI, C.G. et al. Utilização do fósforo na alimentação de bovinos. PUBVET, Londrina, V. 6, N. 17, Ed. 204, Art. 1365, 2012.

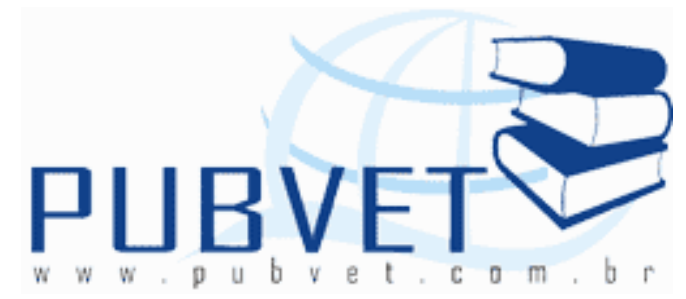

PUBVET, Publicações em Medicina Veterinária e Zootecnia.

\title{
Utilização do fósforo na alimentação de bovinos
}

Carlos Giovani Pancoti ${ }^{1 *}$, Raphael de Castro Mourão ${ }^{1,}$ Gabriela Maldini P.M. Amaral $^{2}$, Thiago Queiroz Resende², Pedro H. A. Carvalho², Andressa Laysse da Silva ${ }^{2}$, Paulo Vitor Valentini ${ }^{3}$

${ }^{1}$ Doutorando em Zootecnia, Departamento de Zootecnia, Escola de Veterinária, Universidade Federal de Minas Gerais, Brasil.

${ }^{2}$ Graduando em Medicina Veterinária, Universidade Federal de Minas Gerais, Brasil.

${ }^{3}$ Mestrando em Zootecnia, Departamento de Zootecnia, Escola de Veterinária, Universidade Federal de Minas Gerais, Brasil.

* E-mail para correspondência: cgpancoti@yahoo.com.br

\section{Resumo}

Nos dias atuais, é crescente a preocupação com a contaminação do ambiente com elementos que se acumulam oriundos de dejetos animais, como o fósforo. Além de acumular no ambiente, o fósforo é o mineral mais caro na suplementação dos animais, seu desperdício gera perda financeira para o produtor. O conhecimento dos requerimentos dos nutrientes acarreta em maior eficiência em todo o sistema. Os autores fazem uma revisão sobre a utilização e a recomendação de fósforo em bovinos.

Palavras-chave: mineral, fósforo, requerimentos, suplementação 
PANCOTI, C.G. et al. Utilização do fósforo na alimentação de bovinos. PUBVET, Londrina, V. 6, N. 17, Ed. 204, Art. 1365, 2012.

\title{
The phosphorus use for cattle feeding
}

\begin{abstract}
There is increasing concern about environmental contamination with elements that build up from animal excretion, such as phosphorus. In addition to accumulating in the environment, phosphorus is the most expensive mineral supplementation for animals, their waste, generates financial loss to the farmer. Knowledge of the requirements of nutrients leads to greater efficiency throughout the system. The authors make a review to recommendation and use of phosphorus in cattle.
\end{abstract}

Keywords: mineral, phosphorus, requirements, supplementation

\section{INTRODUÇÃO}

Nos últimos anos, estudos da FAO indicam que o mundo chegará a 9 bilhões de pessoas em 2050, o que aliado a uma melhor distribuição de renda, aponta para uma previsão de aumento na demanda de alimentos em torno de $70 \%$, como consequencia o aumento do consumo de produtos de origem animal, além da redução das áreas destinadas à exploração pecuária levarem a um aumento da pressão por produção. Exigindo assim do sistema, uma maior eficiência, reduzindo perdas e custos para se enquadrar em um meio cada vez mais competitivo e com margens reais de lucros cada vez menores. Neste ambiente, a mineralização na alimentação animal exerce papel fundamental.

Os minerais, embora presentes em menores proporções do que outros nutrientes da dieta, tais como proteína e a gordura, desempenham funções vitais e suas deficiências acarretam alterações de ordem produtiva, reprodutiva e de saúde. Os minerais possuem basicamente três funções no organismo animal: composição estrutural de órgãos e tecidos, constituintes de tecidos e fluidos corporais e catalisadores de sistemas enzimáticos e hormonais (Underwood, 1981). 
PANCOTI, C.G. et al. Utilização do fósforo na alimentação de bovinos. PUBVET, Londrina, V. 6, N. 17, Ed. 204, Art. 1365, 2012.

O fósforo $(P)$ é o segundo elemento mineral mais abundante no organismo. Cerca de $80 \%$ está presente nos ossos e dentes, além de localizar-se também em todas as células e em quase toda transação envolvendo formação e quebra de ligações de alta energia. O P também está intimamente envolvido no equilíbrio ácido-básico do sangue e de outros fluidos corporais, sendo componente fosfolipídico, fosfoprotéico e dos ácidos nucléicos.

A absorção de $\mathrm{P}$ ocorre principalmente no intestino delgado, e somente pequenas quantidades são absorvidas no rúmen, omaso e abomaso. A homeostase do $\mathrm{P}$ é predominantemente mantida por reciclagem salivar $\mathrm{e}$ excreção fecal, sendo proporcional a quantidade de $\mathrm{P}$ consumida e absorvida (NRC, 2001).

Além disso, o $P$ também é requerido pelos microrganismos ruminais para digestão da celulose e síntese de proteína microbiana (Van Soest, 1994).A recomendação de $P$ disponível no rumen seria de $5 \mathrm{~g} / \mathrm{kg}$ de $\mathrm{MO}$ digestível para otimização da atividade microbiana.

A falta de energia e proteína é, freqüentemente, responsável por níveis subótimos de produção. Todavia, desequilíbrios minerais nos solos e forrageiras vêm sendo responsabilizados pelo baixo desempenho produtivo e reprodutivo de ruminantes sob pastejo em áreas tropicais (McDowell, 1999). Fontes de suplementos minerais de $\mathrm{P}$ (fosfatos monocálcico e bicálcico) são adicionadas acima da recomendação, no intuito de se garantir "margem de segurança", resultando em 25 a 35\% de excesso de $\mathrm{P}$ na dieta e aumento de $30 \%$ na excreção de P (Sutton e Lander, 2003).

As pesquisas realizadas para a adequação dos teores de $\mathrm{P}$ nas dietas têm sido direcionadas principalmente para a redução da excreção desse elemento para o ambiente (Mjoun et al., 2008). Perdas de $P$ na água podem levar ao enriquecimento e à eutrificação, acelerando o crescimento de algas, reduzindo o oxigênio dissolvido e contribuindo para a diminuição da qualidade da água, além de aumentar os custos com a despoluição (Sharpley et al., 1999). 
PANCOTI, C.G. et al. Utilização do fósforo na alimentação de bovinos. PUBVET, Londrina, V. 6, N. 17, Ed. 204, Art. 1365, 2012.

No solo, os íons fosfato facilmente formam compostos com outros minerais. Em baixo potencial hidrogeniônico $(\mathrm{pH})$, o fosfato liga-se aos óxidos de ferro (Fe), alumínio ( $\mathrm{Al}$ ) e manganês $(\mathrm{Mn})$ formando precipitados insolúveis, e com $\mathrm{pH}$ alto, o fosfato liga-se com o cálcio $(\mathrm{Ca})$, tornando-o praticamente indisponível (Barnes et al., 2007).

O objetivo desta revisão é abordar sobre os requerimentos nutricionais de fósforo, sua utilização, e seus efeitos nutricionais e produtivos da sua suplementação para bovinos.

\section{REVISÃO DE LITERATURA}

\subsection{REQUERIMENTOS, DISPONIBILIDADE, ABSORÇÃO E UTILIZAÇÃO DO FÓSFORO EM RUMINANTES}

Atualmente, estimativas do total de $\mathrm{P}$ requerido pelos ruminantes são importantes para otimizar o desempenho e diminuir o risco de excesso de $P$ excretado para o ambiente. O método fatorial é o método mais utilizado para estimar os requerimentos de $P$ para bovinos (ARC, 1980; NRC, 2001). O requerimento de $\mathrm{P}$ absorvido é a soma dos requerimentos para mantença, produção de leite, crescimento e gestação. $O$ total de $P$ dietético requerido é calculado dividindo-se o absorvido pelo coeficiente de absorção do elemento e diferentes ingredientes possuem diferentes coeficientes (NRC, 2001).

No rúmen, a disponibilidade dos minerais e sua utilização dependem da taxa de passagem e da interação com a população microbiana (Van Soest, 1994). As disponibilidades do $\mathrm{Ca}$ e $\mathrm{P}$ podem ser significativamente alteradas em virtude de suas combinações químicas ou associações físicas com outros componentes da dieta. O ácido fítico afeta a absorção intestinal do Ca e $\mathrm{P}$, porém no rúmen, em virtude da produção da fitase microbiana, o fitato é amplamente utilizado (Blaney et al., 1982). A absorção de $P$ também pode ser prejudicada pelo magnésio $(\mathrm{Mg})$, Al e $\mathrm{Fe}$, que formam precipitados, bem como 
PANCOTI, C.G. et al. Utilização do fósforo na alimentação de bovinos. PUBVET, Londrina, V. 6, N. 17, Ed. 204, Art. 1365, 2012.

pelo molibdênio (Mo) e cobre $(\mathrm{Cu})$, que interferem diretamente na sua absorção (McDowell, 1999).

O controle hormonal do metabolismo de cálcio e de fósforo ocorre pela ação do hormônio da paratireóide (PTH, paratormônio) e da calcitonina. O PTH é secretado na glândula paratireóide e aumenta a síntese biológica de 1,25 dihidroxicolecalciferol, que mobiliza cálcio do osso e aumenta a absorção de cálcio no intestino delgado, e também aumenta aconcentração sanguínea de cálcio. A calcitonina é secretada pelas células ultimobraquiais da glândula tireóide e inibe a reabsorção de cálcio dos ossos, diminuindo a concentração de cálcio do sangue. A vitamina D (1,25 dihidroxicolecalciferol ou calcitriol) é indispensável para que o processo ocorra, e este é o motivo pelo qual as deficiências de $\mathrm{Ca}, \mathrm{P}$ e de vitamina $\mathrm{D}$ produzem sinais clínicos semelhantes, ou seja, raquitismo em animais jovens e osteomalácea em animais adultos (NRC, 1989; 1996 e 2001).O sistema de transporte ativo dependente de vitamina D é distinto para o Ca. Porém, baixos teores de $\mathrm{P}$ na dieta podem estimular a vitamina D ativa, resultando em absorção mais eficiente (NRC 2001).

A exigência de vitamina $D$ em bovinos de corte é de $275 \mathrm{UI} / \mathrm{kg}$ de matéria seca (MS) para cada dia (d) (NRC, 2000). Porém, animais que recebem luz solar ou que se alimentam de forrageiras secas ao sol raramente necessitam desta suplementação, a não ser que sejam animais confinados e que recebam dieta conservada (NRC, 2000).

Montgomery et al. (2004) avaliaram o efeito da vitamina D sobre o status de Ca e $\mathrm{P}$ em novilhos Bos taurus e Bos indicus. Os níveis de Ca e P plasmáticos e musculares não foram afetados pelo tipo biológico, indicando que a homeostase desses minerais é fortemente e estritamente regulada nessas espécies.

A concentração plasmática da forma ativa da vitamina $D\left(1,25 \mathrm{OH}_{2} \mathrm{D}\right)$ é menor para o gado europeu, atribuída a uma adaptação genética e ambiental, devido à menor luminosidade nos países da Europa. Porém, mais pesquisas são necessárias para determinar as conseqüências práticas dessas diferenças na 
PANCOTI, C.G. et al. Utilização do fósforo na alimentação de bovinos. PUBVET, Londrina, V. 6, N. 17, Ed. 204, Art. 1365, 2012.

concentração de vitamina $D$ entre espécies antes de preconizar doses distintas de vitamina $D$ para as diferentes raças.

Block et al. (2004) sugeriram que os requisitos de bovinos em terminação com relação ao P, são superestimados pelo NRC (2001) devido a incorretas estimativas nos requerimentos para ganho e mantença, ou pelo coeficiente de absorção. O NRC (2001) relatou coeficiente de absorção médio para o fosfato bicálcico de $75 \%$, comparado com $68 \%$ citado no NRC (1996) para todos os alimentos. Ainda de acordo com o NRC (2001), forragens apresentam reduzido coeficiente de absorção de $\mathrm{P}$ (64\%) quando comparadas com concentrados (70\%). Fatores como espécie, maturidade da planta, clima, o tipo de solo e sua concentração de minerais, não devem ser utilizadas separadamente para predizer a concentração mineral da forrageira (Baker, 1977). Geralmente, a concentração da maioria dos minerais são maiores em leguminosas com relação às gramíneas.

Tabela 1: Concentração de minerais em algumas gramíneas e leguminosas forrageiras

\begin{tabular}{lcccccc}
\hline \multirow{2}{*}{ Espécie } & \multicolumn{7}{c}{ Mineral $(\mathrm{g} / \mathrm{kg})$} \\
\cline { 2 - 7 } & $\mathrm{N}$ & $\mathrm{P}$ & $\mathrm{K}$ & $\mathrm{Ca}$ & $\mathrm{Mg}$ & $\mathrm{S}$ \\
\hline Brachiaria decumbens & 17,0 & 2,6 & 17,7 & 5,3 & 3,4 & 1,4 \\
Panicum maximum & 18,8 & 1,7 & 21,1 & 6,4 & 2,6 & 1,2 \\
Pennisetum purpureum & 23,5 & 2,1 & 24,8 & 4,4 & 2,5 & 1,0 \\
Medicago sativa & 45,1 & 3,9 & 27,0 & 12,6 & 5,9 & 2,5 \\
Stylosanthes guyanensis & 28,1 & 2,4 & 17,5 & 16,2 & 4,8 & 1,6 \\
\hline
\end{tabular}

Fonte: Adaptado de Gallo et al. (1974). N=nitrogênio, $\mathrm{P}=$ fósforo, $\mathrm{K}=$ potássio, $\mathrm{Ca}=$ cálcio, $\mathrm{Mg}=$ magnésio, $\mathrm{S}=$ enxofre.

Com relação à idade da planta (Ramos, 1997), constata-se que a concentração da maioria dos minerais nas forrageiras tendem a decrescer com a idade da planta, e que, para alguns minerais, a influência da maturidade sob suas concentrações é maior, como no caso do nitrogênio e fósforo, os dois de grande importância no planejamento da suplementação dos animais em pastejo. 
PANCOTI, C.G. et al. Utilização do fósforo na alimentação de bovinos. PUBVET, Londrina, V. 6, N. 17, Ed. 204, Art. 1365, 2012.

Tabela 2: Concentrações médias de minerais na forrageira Panicum maximum nos diferentes intervalos de crescimento

\begin{tabular}{ccccccc}
\hline \multirow{2}{*}{ Idade (semanas) } & \multicolumn{7}{c}{ Mineral $(\mathrm{g} / \mathrm{kg})$} \\
\cline { 2 - 7 } & $\mathrm{N}$ & $\mathrm{P}$ & $\mathrm{K}$ & $\mathrm{Ca}$ & $\mathrm{Mg}$ & $\mathrm{S}$ \\
\hline 4 & 39,0 & 3,7 & 31,7 & 4,2 & 2,7 & 2,7 \\
6 & 27,5 & 2,9 & 33,7 & 4,5 & 3,3 & 2,6 \\
8 & 23,0 & 2,6 & 28,8 & 5,2 & 4,1 & 2,6 \\
14 & 12,5 & 1,9 & 22,1 & 5,8 & 3,6 & 2,0 \\
22 & 12,2 & 2,7 & 18,3 & 5,8 & 3,4 & 2,1 \\
\hline
\end{tabular}

Adaptado de Ramos (1997). N=nitrogênio, $\mathrm{P}=$ fósforo, $\mathrm{K}=$ potássio, Ca=cálcio, $\mathrm{Mg}=$ magnésio, $\mathrm{S}=$ enxofre.

Geisert et al. (2010) avaliaram os requerimentos e a excreção de P em 60 novilhas (com peso vivo (PV) médio de $278 \mathrm{~kg}, \pm 17 \mathrm{~kg}$ ). Os tratamentos baseavam-se em diferentes concentrações de $P$ na MS da dieta $(0,10 ; 0,17$; 0,$24 ; 0,31$ e $0,38 \%$ ) que foram balanceadas para $12,5 \%$ de proteína bruta (PB). A fonte de Ca foi o calcário (1,4\% na MS) e de P o fosfato monosódico.

Os valores de consumo, desempenho e características de carcaça, estão demonstrados na tabela 3 .

Tabela 3: Efeito de crescentes concentrações de fósforo na dieta sobre o desempenho e características de carcaça de novilhas

\begin{tabular}{|c|c|c|c|c|c|c|c|}
\hline \multirow{2}{*}{ Item } & \multicolumn{5}{|c|}{ Fósforo (\%MS) } & \multirow[b]{2}{*}{ L } & \multirow{2}{*}{$\mathrm{Q}$} \\
\hline & 0,1 & 0,17 & 0,24 & 0,31 & 0,38 & & \\
\hline $\mathrm{CP} g / \mathrm{d}$ & 7,4 & 14,1 & 20,5 & 25,9 & 29,6 & $<0,01$ & $<0,01$ \\
\hline PV inicial $(\mathrm{kg})$ & 281 & 279 & 280 & 280 & 278 & 0,77 & 0,98 \\
\hline PV final $(\mathrm{kg})$ & 493 & 530 & 528 & 526 & 506 & 0,52 & $<0,01$ \\
\hline CMS kg/d & 7,4 & 8,3 & 8,5 & 8,3 & 7,7 & 0,38 & $<0,01$ \\
\hline GMD kg/d & 1,17 & 1,39 & 1,38 & 1,4 & 1,26 & 0,26 & $<0,01$ \\
\hline Gordura. $(\mathrm{cm})$ & 0,89 & 0,97 & 1,07 & 0,86 & 0,99 & 0,76 & 0,42 \\
\hline Longíssimus $\left(\mathrm{cm}^{2}\right)$ & 79,8 & 78,0 & 76,0 & 78,1 & 72,6 & 0,11 & 0,76 \\
\hline Marmoreio & 486 & 565 & 529 & 546 & 554 & 0,1 & 0,28 \\
\hline
\end{tabular}

Fonte: Adaptado de Geisert et al. (2010); MS=matéria seca; $L=$ efeito linear; $\mathrm{Q}=$ efeito quadrático; $\mathrm{CP}=$ consumo de fósforo; $\mathrm{PV}=$ peso vivo; $\mathrm{CMS}=$ consumo de matéria seca; $\mathrm{GMD}=$ ganho médio diário.

Ocorreu tanto efeito tanto linear quanto quadrático no consumo (C) de P. Houve efeito quadrático $(p<0,01)$ para ganho de peso e no CMS. Animais em 
PANCOTI, C.G. et al. Utilização do fósforo na alimentação de bovinos. PUBVET, Londrina, V. 6, N. 17, Ed. 204, Art. 1365, 2012.

dietas com 0,10 e $0,38 \%$ de $\mathrm{P}$ tiveram menores CMS e consequentemente menor ganho de peso. O menor consumo verificado no tratamento com $0,38 \%$ de $P$ foi provavelmente devido à diminuição da palatabilidade do fosfato, enquanto no tratamento com $0,10 \%$ de $P$, esta inferioridade ocorreu em função do baixo consumo de $P$, pois de acordo com Van Soest (1994), há diminuição do CMS em dietas com deficiência de P. Os dados sugeriram que concentrações de $0,17 \%$ de $P$ são adequadas para manter o CMS e ganho de peso. Erickson et al. (2002) também relataram concentração de $0,16 \%$ de $P$ como ótimo para terminação em novilhos.

O teor de Ca nas dietas foi constante $(0,70 \% \mathrm{Ca})$, com relações de Ca:P variando entre $1,8: 1$ e 7:1, sendo que, segundo os autores, seria improvável que essa relação tenha afetado o CMS ou o ganho de peso.

Não houve diferença na concentração plasmática de $\mathrm{P}$ entre os tratamentos no primeiro dia, com concentração média de $7,12 \mathrm{mg} / \mathrm{dL}$. Concentração plasmática menor que $4,5 \mathrm{mg} / \mathrm{dL}$ é sinal de deficiência de $\mathrm{P}$ sendo que a concentração deve se manter entre 4,5 a $5 \mathrm{mg} / \mathrm{dl}$ (Ternouth et al., 1996).

As concentrações para o tratamento $0,17 \%$ de $P$ entre os dias 0 e 180 foram semelhantes $(p>0,01)$, indicando que não houve sinal de deficiência de $P$. Porém, na concentração de $P$ de 0,10 , esta foi menor $(p>0,01)$ entre os dias 30 a 60 e entre 150 a 180 dias, evidenciando que houve deficiência de $P$ na dieta nesta concentração. No período entre 90 a 120 dias, não houve diferença $(p>0,01)$, indicando que houve homeostase por reabsorção óssea. A concentração de $P$ não afetou a mensuração física dos ossos da falange $\mathrm{e}$ metacarpo (180 dias de avaliação). Houve aumento linear $(p<0,01)$ do peso das cinzas das falanges com o aumento do $P$ na dieta, mas essa significância não foi relatada nos ossos metacárpicos. Shupe et al. (1988) e Ternouth (1990) relataram que os ossos metacárpicos são pobres como indicadores do status mineral. Erickson et al. (2002) não encontraram diferenças nos pesos das cinzas entre falanges $(28,7 \mathrm{~g})$ e entre metacarpos $(238 \mathrm{~g})$ em bezerros alimentados com dietas entre 0,16 a $0,40 \% \mathrm{P}$ ( $\%$ na MS). 
PANCOTI, C.G. et al. Utilização do fósforo na alimentação de bovinos. PUBVET, Londrina, V. 6, N. 17, Ed. 204, Art. 1365, 2012.

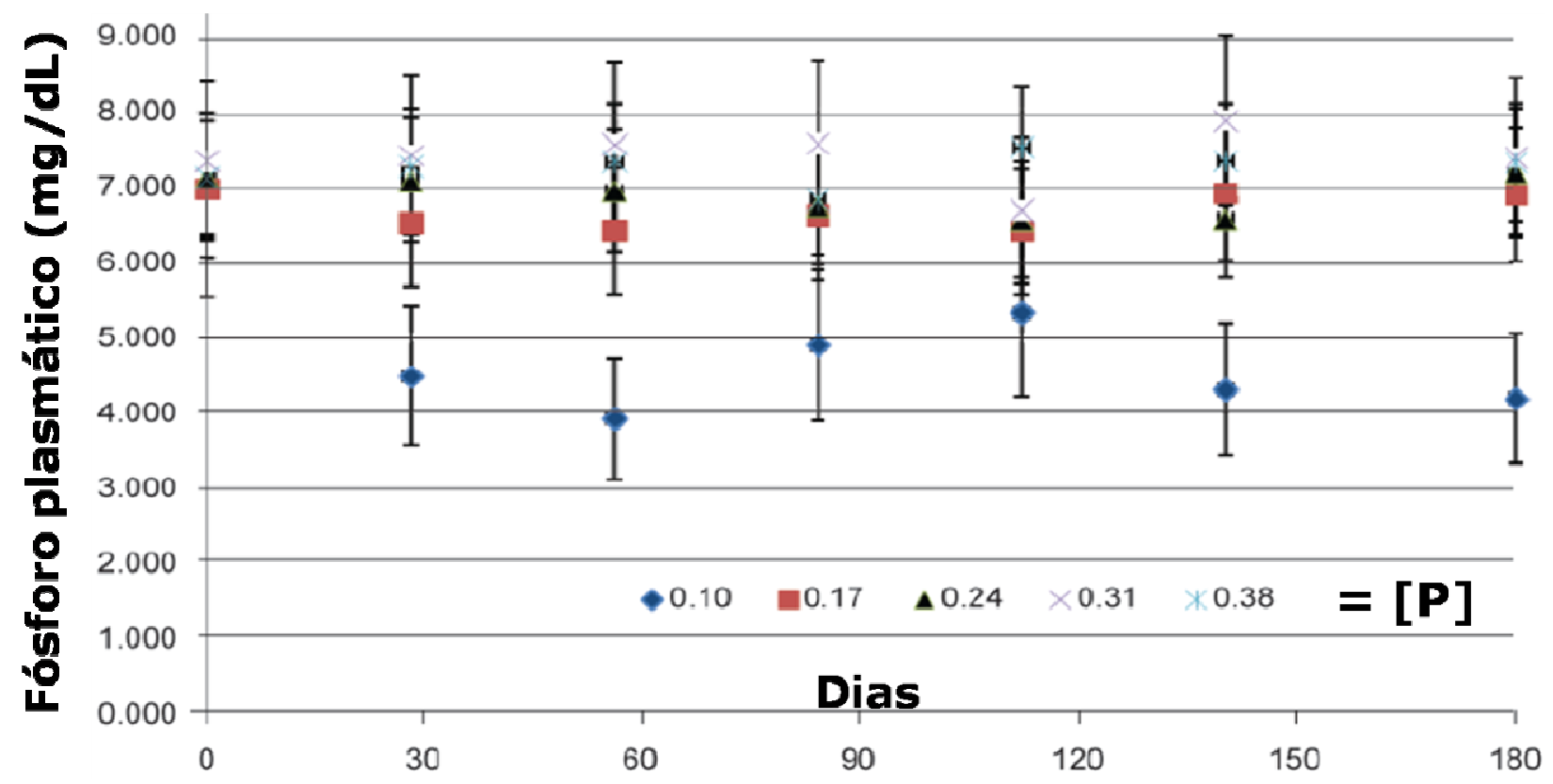

Figura 1: Concentração plasmática de fósforo ([P]) em novilhas alimentadas com diferentes teores de fósforo na dieta. Fonte: Adaptado de Geisert et al. (2010).

O NRC (1996) sugeriu que novilhas com ganho médio diário (GMD) de 1,32kg e PV entre 278 e $573 \mathrm{~kg}$, requerem $21 \mathrm{~g} \mathrm{P} /$ dia com coeficiente de absorção do $\mathrm{P}$ de $68 \%$.

No estudo de Geisert et al. (2010), a recomendação de $P$ foi de $14,1 \mathrm{~g} / \mathrm{d}$, sem efeito adverso na concentração plasmática de $\mathrm{P}$, desempenho ou crescimento ósseo. Corroborado por Erickson et al. (2002) que relataram que a exigência de $\mathrm{P}$ para bezerros deve ser menor que 14,2g P/dia e abaixo de 15,5g P/dia para novilhos com um ano de idade. Isso sugere que as exigências para terminação recomendado pelo NRC (1996) seja menor, provavelmente por falha na estimativa do coeficiente de absorção, além de não se contabilizar a reciclagem de $\mathrm{P}$ pela saliva.

Estes mesmos autores também não encontraram diferenças no CMS $(8,84 \mathrm{~kg} / \mathrm{dia}), \operatorname{GMD}(1,5 \mathrm{~kg})$, espessura de gordura de cobertura $(1,15 \mathrm{~cm})$, área do longíssimus dorsi $\left(108,3 \mathrm{~cm}^{2}\right)$ e grau de marmoreio (543) em novilhos mestiços alimentados com cinco concentrações de $P(0,16 ; 0,22 ; 0,28 ; 0,34$ e 
PANCOTI, C.G. et al. Utilização do fósforo na alimentação de bovinos. PUBVET, Londrina, V. 6, N. 17, Ed. 204, Art. 1365, 2012.

$0,40 \% \mathrm{MS})$. No dia zero, a média do $P$ plasmático foi de $7,5 \mathrm{mg} / \mathrm{dL},(p>0,05)$. Pesquisas apontam concentrações plasmáticas de $P$ entre 4,5 e 5,0mg/dL (Ternouth et al., 1996). O tratamento com 0,16\% de $\mathrm{P}$ apresentou durante $\mathrm{O}$ experimento, concentração plasmática média de $P$ de $5,0 \mathrm{mg} / \mathrm{dL}$. $O$ tratamento $0,10 \%$ de $P$ apresentou diminuição $(p<0,01)$ na concentração plasmática entre os dias 30 a 60 e 150 a 180, mostrando que houve deficiência de $P$ para este tratamento.

Ezequiel (1987) encontrou valor médio de $17,6 \mathrm{mg} / \mathrm{kg}$ PV na excreção metabólica diária de $\mathrm{P}$ em novilhas Nelore em crescimento. Valadares Filho et al., (2010) - BR-Corte, sugeriram a adoção do valor obtido por Ezequiel (1987) de 17,6mg/kg PV para as exigências diárias de $P$ para mantença, por ter sido obtido em condições brasileiras.

Clark et al. (2007) avaliaram o efeito de 20 e 10\% de restrição do CMS em novilhos de corte sobre a retenção de $\mathrm{P}$. Os benefícios associados à restrição do CMS incluem o aumento da digestibilidade, da eficiência alimentar, na diminuição dos custos e no aumento do GMD (Schmidt et al., 2005). Porém, quando os consumos de MS e energia foram diminuídos concomitantemente, houve redução do GMD e da qualidade da carcaça. A restrição do CMS sem afetar o consumo de energia e proteína, promovem aumento do GMD e da qualidade da carcaça (Schmidt et al., 2005).

Tabela 4: Efeito de dietas formuladas para GMD (ganho médio diário) de $1,6 \mathrm{~kg} / \mathrm{dia}$, quando ocorrido 100 (AL), 90 (IR90) ou $80 \%$ (IR80) do CMS ad libitum na retenção de fósforo $(P)$ em novilhos mestiços de corte

\begin{tabular}{lcccc}
\hline \multirow{2}{*}{ Item } & \multicolumn{3}{c}{ Tratamento } & \multirow{2}{*}{ Valor de $\mathrm{p}$} \\
\cline { 2 - 4 } & $\mathrm{AL}$ & IR90 & IR80 & \\
\hline CP g/d & 58,6 & 58,9 & 56,0 & $>0,47$ \\
P fecal (\% CP) & 55,1 & 52,0 & 47,4 & $>0,29$ \\
P urina (\% CP) & 10,7 & 17,8 & 18,6 & $>0,11$ \\
P retido g/d & 20,3 & 18,0 & 19,3 & $>0,57$ \\
P retido (\% CP) & 34,2 & 30,2 & 34,0 & $>0,46$ \\
\hline
\end{tabular}

Fonte: Adaptado de Clark et al. (2007). $\mathrm{CP}=$ consumo de fósforo; $\mathrm{AL}=\mathrm{ad}$ libitum; IR90=ingestão restrita a 90\%; IR80=ingestão restrita a $80 \%$. 
PANCOTI, C.G. et al. Utilização do fósforo na alimentação de bovinos. PUBVET, Londrina, V. 6, N. 17, Ed. 204, Art. 1365, 2012.

Os autores não observaram nenhum efeito sobre os parâmetros de consumo, excreção fecal e urinária e retenção do $P$ com relação aos tratamentos, e concluíram que esta estratégia poderia ser utilizada efetivamente. Houve diminuição no consumo e da excreção de MS, e aumento da digestibilidade com a restrição do fornecimento de MS. Porém, similar consumo de proteína e energia foram mantidos entre os tratamentos.

A excreção de $P$ está relacionada com o consumo de $P$ (Meyer et al., 2005). Block et al. (2004) relataram elevada excreção de $P$ em dietas com elevada concentração de $\mathrm{P}$ ou de concentrado. Independente da quantidade de $\mathrm{P}$ consumido, os animais excretam mais $\mathrm{P}$ urinário em dietas com concentrado, em relação a dietas de forrageiras. Morse et al. (1992) e Block et al. (2004) relatam que o $P$ é excretado na urina após os requerimentos de mantença $\mathrm{e}$ produção serem atingidos, ou quando o mecanismo de excreção de $\mathrm{P}$ salivar está saturado. Perda fecal de $\mathrm{P}$ ocorre quando há excesso de $\mathrm{P}$ na dieta e na forma endógena.

Respostas à suplementação de $\mathrm{P}$ em bovinos alimentados com dietas deficientes em $P$ podem ser confundidas com limitação de proteína, pois a suplementação com $P$ não afeta o ganho de peso quando o $N$ é limitante (Brokman et al., 2008).

Vasconcelos et al. (2009) avaliaram o efeito das concentrações de proteína bruta (PB) e uréia sobre a utilização do $\mathrm{P}$ em bovinos de corte. $\mathrm{O}$ consumo de $P$, bem como a excreção urinária e fecal de $P$ aumentaram com a elevação da PB da dieta. Não foram observadas diferenças na absorção aparente do P e P retido com o aumento da PB. Porém, aumentos na proporção de PB da dieta pela adição de uréia, diminuíram o consumo de $\mathrm{P}$, resultando em menor excreção de $P$. 
PANCOTI, C.G. et al. Utilização do fósforo na alimentação de bovinos. PUBVET, Londrina, V. 6, N. 17, Ed. 204, Art. 1365, 2012.

Tabela 5: Valores médios de produção e composição de leite de vacas Holandês (até os 165d de lactação) recebendo concentrações de 0,37 e 0,57 (\% matéria seca) de fósforo $(P)$ na dieta total

\begin{tabular}{lccc}
\hline Item & $0,37 \%$ & $0,57 \%$ & Valor de P \\
\hline Produção leite $(\mathrm{kg} / \mathrm{d})$ & 35,1 & 34,9 & 0,81 \\
LCG $(\mathrm{kg} / \mathrm{d})$ & 36,8 & 36,9 & 0,87 \\
Gordura $(\%)$ & 3,92 & 3,98 & 0,32 \\
Proteína $(\%)$ & 2,9 & 2,91 & 0,82 \\
CCS $\left(10^{3} / \mathrm{ml}\right)$ & 319 & 366 & 0,39 \\
\hline
\end{tabular}

Fonte: Adaptado de Lopez et al. (2004). LCG=leite corrigido para gordura $(3,5 \%) ; C C S=$ contagem de células somáticas.

López et al. (2004) avaliaram parâmetros de produção e composição do leite e de saúde, em vacas alimentadas com 0,37 e 0,57\% P na MS. Os autores não encontraram diferença na produção (35kg/dia), na gordura $(3,95 \%)$, na proteína $(2,90 \%)$ e na contagem de células somáticas - CCS (342.000 células $/ \mathrm{mL}$ ) do leite.

A concentração plasmática do $P$ na dieta com $0,37 \%$ de $P$ foi de 6,1 e $6,2 \mathrm{mg} / \mathrm{dL}$ e valores de 6,8 e $6,9 \mathrm{mg} / \mathrm{dL}$ para $0,57 \% \mathrm{P}$ na dieta, no $50^{\circ}$ e $100^{\circ}$ dias em lactação (DEL) respectivamente. Valores considerados normais para vacas em lactação estariam entre 4,4 a 9,2mg/dL (Forar et al., 1982). Wu et al. (2001) encontraram valores de 6,1 e $6,5 \mathrm{mg} / \mathrm{dL}$ em vacas alimentadas com 0,39 e $0,47 \%$ P. Logo, o fornecimento de $\mathrm{P}$ acima das exigências $(0,57 \%)$ não provocou nenhum benefício para os animais.

Ao avaliarem parâmetros de saúde nas vacas leiteiras, Lopez et al. (2004) não observaram efeito da suplementação acima dos requerimentos. Não houve relação entre menor ocorrência de doenças e aumento na suplementação de fósforo 
PANCOTI, C.G. et al. Utilização do fósforo na alimentação de bovinos. PUBVET, Londrina, V. 6, N. 17, Ed. 204, Art. 1365, 2012.

Tabela 6: Ocorrência de problemas de saúde na fase de lactação de vacas Holandês recebendo concentrações de 0,37 e 0,57 (\% matéria seca) de fósforo (P) na dieta total

\begin{tabular}{lccc}
\hline Item & $0,37 \% \mathrm{P}$ & $0,57 \% \mathrm{P}$ & Valor de P \\
\hline Mastite & 44 & 47 & 0,73 \\
Problema de casco & 24 & 27 & 0,66 \\
Retenção de placenta & 21 & 14 & 0,19 \\
Piometra & 8 & 10 & 0,76 \\
Deslocamento de abomaso & 8 & 8 & 0,99 \\
Edema de úbere & 2 & 6 & 0,28 \\
Diarréia & 2 & 5 & 0,45 \\
Cetose & 2 & 3 & 0,99 \\
Infecção respiratória & 2 & 2 & 0,99 \\
Febre do leite & 3 & 0 & 0,12 \\
Distocia & 1 & 0 & 0,50 \\
\hline Fonte:
\end{tabular}

Fonte: Adaptado de Lopez et al. (2004).

Wu e Satter (1999) compararam dietas com ou sem suplementação de $P$, contendo 0,38 e 0,48\% de $P$, não encontrando diferença na produção de leite e no desempenho reprodutivo. A produção de leite sob curtos períodos pode não ser um bom indicador do status de $\mathrm{P}$ devido à mobilização óssea, ocorrendo principalmente no início da lactação. Logo, Wu et al. (2000) avaliaram então o status do P durante toda a lactação, desde o parto até a secagem. Utilizaram 26 vacas multíparas por 308 dias em lactação, avaliaram dietas com 0,31; 0,40 e $0,49 \%$ de $P$ (fosfato monosódico para as duas últimas). As dietas eram à base de silagem de alfafa (30\%), silagem de milho (20\%), milho, soja e polpa cítrica. As dietas eram isoenergéticas, isoprotéicas e isofibrosas. O Ca foi o mesmo entre os tratamentos. O CMS foi similar entre tratamentos, assim como a produção de leite. A curva de lactação foi aproximadamente constante por 20 semanas após o pico de produção, porém, houve interação entre tratamento e mês de lactação $(p<0,01)$, refletindo na menor produção de leite para baixo teor de $\mathrm{P}$ por volta da $25^{\circ}$ semana, cujo valor foi, em média, $3,3 \mathrm{~kg} /$ dia menor. 


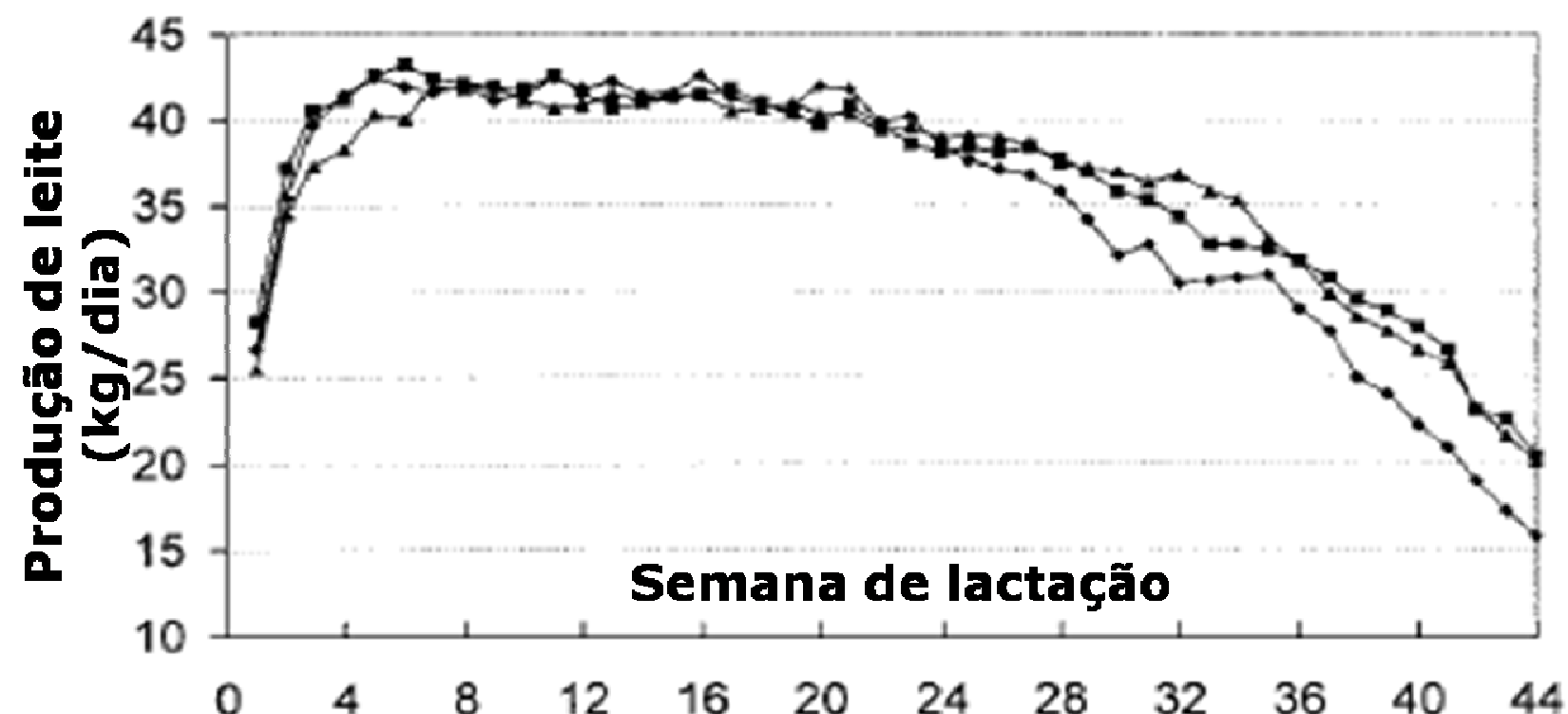

Figura 2: Curvas de lactação de vacas alimentadas com diferentes concentrações $(\%)$ de fósforo $(\bullet=0,31 ; \quad \cdot=0,40 ; \quad \boldsymbol{\Lambda}=0,49)$ na dieta (Adaptado de Wu et al., 2000).

Não houve diferença na composição do leite, embora tenha ocorrido algumas interações. As concentrações de $\mathrm{P}$ no plasma e no leite não foram relacionadas $\left(r^{2}=0,02\right)$. Porém, alguma relação existe entre concentração de $P$ e proteína no leite $\left(r^{2}=0,37\right)$. Segundo Jenness $(1985)$, a metade do $\mathrm{P}$ no leite está complexada com a caseína e a outra parte difundida na forma iônica.

Wu e Satter (2000), realizaram uma revisão dos últimos 30 anos (envolvendo 785 vacas) da utilização de fósforo acima das recomendações, sobre parâmetros reprodutivos.

Tabela 7: Avaliação da suplementação de fósforo (P) acima dos requerimentos sobre parâmetros reprodutivos

\begin{tabular}{lcc}
\hline Item & Baixo P & Alto P \\
\hline Dias para $1^{\circ}$ estro & 46 & 48 \\
Dias para $1^{\text {a }}$ inseminação & 73 & 76 \\
Serviços/Concepção & 1,8 & 1,9 \\
Taxa de Gestação \% & 87 & 86 \\
\hline
\end{tabular}

Fonte: Adaptado de Wu e Satter (2000) 
PANCOTI, C.G. et al. Utilização do fósforo na alimentação de bovinos. PUBVET, Londrina, V. 6, N. 17, Ed. 204, Art. 1365, 2012.

Os autores concluíram que não houve benefício com a suplementação de fósforo acima de $0,40 \%$.

Dias et al. (2011), trabalhando com ovinos em crescimento, encontraram que o excesso de $P$ na dieta aumentou a concentração de $P$ no plasma, e que houve relação inversa entre retenção de $P$ no osso e $P$ excretado na urina, indicando que o $\mathrm{P}$ mobilizado e não utilizado pelos tecidos moles foi excretado na urina e nas fezes. Os autores concluíram que a suplementação de $P$ frequentemente representa um desnecessário custo alimentar e dano potencial ao ambiente, devido não somente ao aumento da excreção de $P$ não absorvido, mas também ao aumento da excreção de $\mathrm{P}$ endógeno.

\section{CONSIDERAÇÕES FINAIS}

O entendimento dos processos que envolvem a utilização dos minerais pelo ruminante, particularmente o fósforo, é importante, de modo a possibilitar o desenvolvimento de estratégias que possam adequar o suprimento de misturas minerais para o animal, garantindo maior vantagem produtiva e econômica, conciliada a uma menor excreção de fósforo, favorecendo uma maior eficiência de utilização desse mineral.

De acordo com a literatura consultada, os valores de exigências do $\mathrm{P}$ para bovinos estão superestimados, ocasionando um gasto desnecessário para um melhor desempenho dos animais e menor excreção fosfatada no ambiente. Os dados avaliados não sugerem que $\mathrm{o}$ aumento de $\mathrm{P}$ na dieta melhore $\mathrm{O}$ desempenho produtivo e reprodutivo. Deve-se avaliar o balanceamento da dieta como um todo e eliminar exageros, pois os custos desses excessos certamente pesam no orçamento, além de apresentar restrições ambientais.

Novas pesquisas sobre a utilização, excreção e requerimentos de $P$ são necessárias, de modo a elucidar o uso eficiente dos nutrientes, especificamente do $P$, principalmente em trabalhos que envolvam condições brasileiras. 


\section{REFERENCIAS BIBLIOGRÁFICAS}

AGRICULTURAL RESEARCH COUNCIL - ARC. The Nutrient Requirements of Ruminant Livestock. London, 1980. 351p.

BARNES, R.F.; NELSON, C.J.; MOORE, K.J.; COLLINS, M.Forages - The Science of Grassland Agriculture. Blackwell Publishing. v.2. 6.ed. 2007. 791p.

BLANEY, J.; GARTNER, J.W.; HEAD, T.A. The effects of oxalate in tropical grasses on calcium, phosphorus and magnesium availability to cattle. J. Agric. Sci., v.99, n.3, p.533-539, 1982.

BLOCK, H.C.; ERICKSON, G.E.; KLOPFENSTEIN, T.J. Re-evaluation of phosphorus requirements and phosphorus retention of feedlot cattle. A review. Prof. Anim. Sci., v.20, p.319-329, 2004.

BROKMAN, A.M.; LEHMKUHLER, J.W.; UNDERSANDER, D.J. Reducing phosphorus inputs for grazing Holstein steers. J. Anim. Sci., v.86, p.712-719, 2007.

CLARK, J.H.; OLSON, K.C.; SCHMIDT, T.B.; LINVILLE, M.L.; ALKIRE, D.O.; MEYER, D.L.; RENTFROW, G.K.; CARR, C.C.; BERG, E.P. Effects of dry matter intake restriction on diet digestion, energy partitioning, phosphorus retention, and ruminal fermentation by beef steers.

J. Anim. Sci., v.85, p.3383-3390, 2007.

DIAS, R.S.; LÓPEZ, S.; PATIÑO, R.M.P.; SILVA, T.S.; SILVA FILHO, J.C.; VITTI, D.M.S.S.; PEÇANHA, M.R.S.R.; KEBREAB, E.; FRANCE, J. An extended model of phosphorus metabolism in growing ruminants. J. Anim. Sci., published online July 15, 2011.

ERICKSON, G.E.; KLOPFENSTEIN, T.J.; MILTON, C.T.; BRINK, D.; ORTH, M.W.; WHITTET., K.M. Phosphorus requirement of finishing feedlot calves. J. Anim. Sci., v.80, p.1690-1695, 2002.

EZEQUIEL, J.M.B. Exigências de proteína e minerais de bovídeos: Frações endógenas. 1987. 131f. Tese (Doutorado em Zootecnia) - Universidade Federal de Viçosa, Viçosa, MG.

FAO. Food Outlook. Global Market Analysis, 2008.

FORAR, F.L.; KINCAID, R.L.; PRESTON, R.L.; HILLERS, J.K. Variation of inorganic phosphorus in blood plasma and milk of lactating cows. J. Dairy Sci., v.65, p.760-763, 1982.

GEISERT, B.G.; ERICKSON, G.E.; KLOPFENSTEIN, T.J.; MACKEN, C.N.; LUEBBE, M.K.; MACDONALD, J.C. Phosphorus requirement and excretion of finishing beef cattle fed different concentrations of phosphorus. J. Anim. Sci., v.88, p.2393-2402, 2010.

JENNESS, R. Biochemical and nutritional aspects of milk and colostrum. The Iowa State University. p.164-197, 1985.

LOPEZ, H.; KANITZ, F.D.; MOREIRA, V.R.; WILTBANK, M.C.; SATTER, L.D. Effect of Dietary Phosphorus on Performance of Lactating Dairy Cows: Milk Production and Cow Health. J. Dairy Sci., v.87, p.139-145, 2004.

McDOWELL, L. R. Minerais para Ruminantes em Regiões Tropicais, Enfatizando o Brasil. University of Florida. 3.ed. 1999. 92p.

MEYER, N.; TRENKLE, A.; PINGEL, D.; BARRETT, K. Phosphorus metabolism in steers fed high grain diets. J. Anim. Sci., v.83(Suppl. 1), p.55. (Abstract). 2005.

MJOUN, K.; KALSCHEUR, K.F.; HIPPEN, A.R.; SCHINGOETHE, D.J. Ruminal Phosphorus Disappearance from Corn and Soybean Feedstuffs. J. Dairy Sci., v.91, p.3938-3946, 2008.

MONTGOMERY, J.L.; BLANTON, J.R..; HORST, R.L.; GALYEAN, M.L.; MORROW, K.J.; WESTER, D.B.; MILLER, M.F. Effects of biological type of beef steers on vitamin D, calcium, and phosphorus status. J. Anim. Sci., v.82, p.2043-2049, 2004. 
MORSE, D.; HEAD, H.H.; WILCOX, C.J.; VAN HORN, H.H.; HISSEM, C.D.; HARRIS JR, B. Effects of concentration of dietary phosphorus an amount and route of excretion. J. Dairy Sci., v.75, p.3039-3049, 1992.

NATIONAL RESEARCH COUNCIL - NRC. Nutrients requirements of beef cattle. 7.ed. Washington, D.C.: 1996. 244p.

NATIONAL RESEARCH COUNCIL - NRC. Nutrients requirements of beef cattle. 7.ed. Washington, D.C.: 2000. 242p.

NATIONAL RESEARCH COUNCIL - NRC. Nutrients requirements of dairy cattle. 7.ed. Washington, D.C.: 2001. 362p.

SHUPE, J.L.; BUTCHER, J.E.; CALL, J.W.; OLSON, A.E.; BLAKE, J.T. Clinical signs of bone changes associated with phosphorus deficiency in beef cattle. Am. J. Vet. Res., v.49, p.1629$1636,1988$.

SCHMIDT, T.B.; OLSON, K.C.; LINVILLE, M.L.; CLARK, J.H.; MEYER, D.L.; BRANDT, M.M.; STAHL, C.A.; RENTFROW, G.K.; BERG, E.P. Effects of dry matter intake restriction on growth performance and carcass merit of finishing steers. Prof. Anim. Sci., v.21, p.332-338, 2005.

SHARPLEY, A.N.; DANIEL, T.; SIMS, T.; LEMUNYON, J.; STEVENS, R.; PARRY, R. Agricultural phosphorus and eutrophication. U.S. Department of Agriculture. Agricultural Research Service. p.42, 1999.

SUTTON, A.; LANDER, C.H.Feed and Animal Management for Dairy Cattle. Nutrient Management Technical Note USDA. n.5, 2003.

TERNOUTH, J.H.; BORTOLUSSI, G.; COATES, D.B.; HENDRICKSEN, R.E.; MCLEAN, R.W. The phosphorus requirements of growing cattle consuming forage diets. J. Agric. Sci., v.126, p.503-510, 1996.

TERNOUTH, J.H. Phosphorus and beef production in northern Australia. Phosphorus in cattle. A review. Trop. Grassl., v.24, p.159-169, 1990.

UNDERWOOD, E.J. The mineral nutrition of livestock. London: Academic Press, 1981. $111 \mathrm{p}$.

VALADARES FILHO, S.C.; MARCONDES, M.I.; CHIZZOTTI, M.L.; PAULINO, P.V.R.Exigências Nutricionais de Zebuínos Puros e Cruzados: BR-Corte. 2.ed. Viçosa, MG. Editora UFV, 2010. 193p.

VAN SOEST, P.J. Nutritional Ecology of the Ruminant. Cornell University Press, Ithaca, NY. 1994.

VASCONCELOS, J.T.; COLE, N.A.; MCBRIDE, K.W.; GUEYE, A.; GALYEAN, M.L.; RICHARDSON, C.R.; GREENE, L.W. Effects of dietary crude protein and supplemental urea levels on nitrogen and phosphorus utilization by feedlot cattle. J. Anim. Sci., v.87, p.1174-1183, 2009.

WU, Z.; SATTER, L.D.; BLOHOWIAK, A.J.; STAUFFACHER, R.H.; WILSON, J.H. Milk production, estimated phosphorus excretion, and bone characteristics of dairy cows fed different amounts of phosphorus for two or three years. J. Dairy Sci., v.84, p.1738-1748, 2001.

WU, Z.; SATTER. L.D. Milk production and reproductive performance of dairy cows fed two levels of phosphorus for two years. J. Dairy Sci., v.83, p.1052-1063, 1999.

WU, Z.; SATTER, L.D.; SOJO, R. Milk production, reproductive performance, and fecal excretion of phosphorus in dairy cows fed three amount of phosphorus. J. Dairy Sci. v.83, p.1028-1041, 2000. 